\title{
Biological microsystem for measuring uric acid in biological fluids
}

\author{
G. Minas ${ }^{\text {a }}{ }^{*}$, J.S. Martins ${ }^{\text {a }}$, J.C. Ribeiro ${ }^{\text {a }}$, R.F. Wolffenbuttel ${ }^{\mathrm{b}}$, J.H. Correia ${ }^{\mathrm{a}}$ \\ a Department of Industrial Electronics, University of Minho, Campus de Azurém, 4800-058 Guimarães, Portugal \\ b Fac. ITS Dept. Microelectronics, Delft University of Technology, Mekelweg 4, 2628 CD Delft, The Netherlands
}

Received 25 September 2002; received in revised form 26 September 2003; accepted 10 October 2003

\begin{abstract}
This paper describes a biological microsystem (bio-system) for measuring uric acid concentration in serum, plasma or urine. Its operation is based on optical absorption in a well-defined part of the visible spectrum. The bio-system is composed by two dies: one is fabricated in polystyrene and contains the microchannels and the other is fabricated in a CMOS standard process and contains the photodetector and readout electronics. The uric acid concentration is measured by using a mixture of $14 \mu \mathrm{l}$ of infinity ${ }^{\mathrm{TM}}$ uric acid reagent with $0.25 \mu l$ of sample. The achieved sensitivity is $0.33 \mathrm{mg} / \mathrm{dl}( \pm 0.6 \%$ of the value in urine of a healthy person), with a $1 \mathrm{~mm}$ lightpath. Using an optical absorption method, a maximum peak at wavelength $\lambda=494 \mathrm{~nm}$, is detected. This bio-system can be included in the group of low-cost disposable devices for biological fluids analysis.
\end{abstract}

(c) 2003 Elsevier B.V. All rights reserved.

Keywords: Bio-system; Uric acid; Optical absorption

\section{Introduction}

Over the past decade, the miniaturization of fluidic analyses systems has become a highly visible and dominant trend in physical and biological sciences [1]. Presently, disease prevention and treatment is often based on the measurement of chemical parameters in biological samples, such as blood and urine. In most cases, the samples need to be sent to a central laboratory for analysis, and the results of routine tests become available after several hours, sometimes days. Apart from the time delay, also the mistakes in the logistics, such as lost samples and mislabeling, may hamper timely diagnosis. Therefore, development in miniaturization of fluidic analyses systems has been driven by a need for rapid, on-line measurements with low concentrations and low samples volumes, which is essential within fields such as DNA analysis, drug discovery, pharmaceutical screening, medical diagnostics, environmental analysis and chemical production. The advantages associated with shrinking analytical systems include improved efficiency with respect to sample size, response times, costs, analytical performance, integration, throughput, automation, and laboratory safety [2]. Keywords in this field are "bio-system", "lab-on-a-chip" or

\footnotetext{
* Corresponding author. Tel.: +351-253510190; fax: +351-253510189. E-mail address: gminas@dei.uminho.pt (G. Minas).
}

" $\mu$ TAS" (micro total analysis system), where macroscopic analysis methods are being miniaturized.

Microscale fluidic systems have distinctive properties as a result of their small dimensions. First of all, liquid flow is generally laminar, not turbulent. Secondly, diffusion in narrow channels is practically the only process for mixing fluids, and thirdly, particles can also be separated by diffusion according to their size [3]. In system signal processing and data pre-handling, the small size and low power consumption of such highly integrated systems could make them highly portable and thus suitable for in situ measurements tasks. The application of the particular bio-system presented here is the measurement of uric acid concentration in human being's urine.

\subsection{Background of the uric acid analysis}

Uric acid is a metabolite of purines, nucleic acids and nucleoproteins. Consequently, abnormal levels may be indicative of a disorder in the metabolism of these substances. Hyperuricaemia may be observed in renal dysfunction, gout, leukemia, polycythaemia, atherosclerosis, diabetes, hypothyroidism, or in some genetic diseases. Decreased levels are present in patients with Wilson's disease. A normal adult synthesizes $26.9-53.8 \mathrm{mg} / \mathrm{dl}$ of uric acid in urine. In case of disease, these values can be as low as $17 \mathrm{mg} / \mathrm{dl}$ or too high up to $67 \mathrm{mg} / \mathrm{dl}$ [4]. 


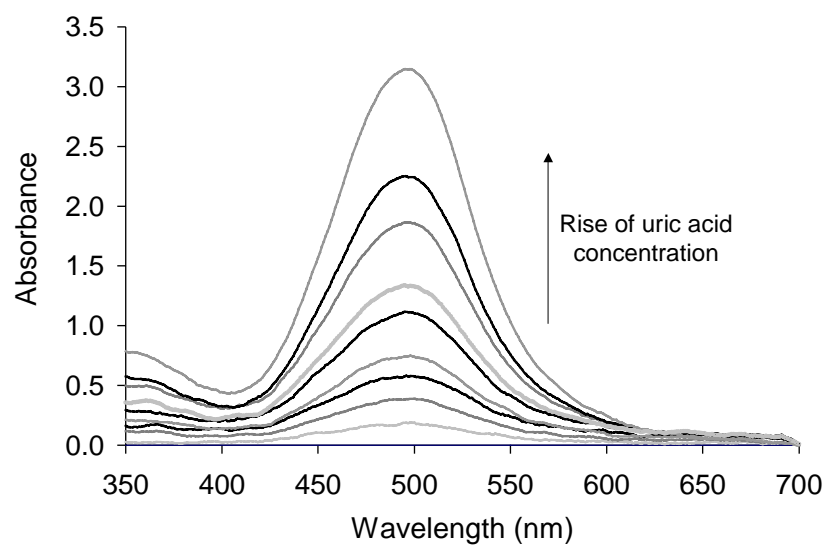

Fig. 1. Macroscopic measurements of absorbance spectra for different uric acid concentrations. From bottom to top curve: 5, 10, 15, 20, 30, $40,60,80$ and $120 \mathrm{mg} / \mathrm{dl}$.

\section{Bio-system design}

The starting point for the design of the bio-system was macroscopic measurements on well-known uric acid standards. These were performed in a $1 \mathrm{~cm}$ lightpath cuvette with a model UV-3101PC SHIMADZU spectrophotometer (Fig. 1). The following conclusions can be drawn from these measurements: (1) the intensity of the color produced by the mixture is directly proportional to the uric acid concentration; (2) the solutions present a linear behavior for concentrations as large as $30 \mathrm{mg} / \mathrm{dl}$ (for higher concentrations the sample should be diluted and re-assayed multiplying the result by the dilute factor); (3) the absorption spectra shows a maximum peak at the wavelength $\lambda=494 \mathrm{~nm}$, with a full-width-half-maximum (FWHM) of $90 \mathrm{~nm}$. At $360 \mathrm{~nm}$ the absorption spectra also shows different absorbance values according to the uric acid concentration. However, the absorbance difference between successive measured concentrations is significantly smaller. In addition the sensitivity is three times less as that at $494 \mathrm{~nm}$.

The bio-system is composed of two dies: one contains the microchannels to carry chemical reagents and samples, and the other contains the uric acid concentration detection system.

\subsection{Polystyrene die}

Fig. 2 shows the design of the bio-system polystyrene die. It is composed of two polystyrene $1 \mathrm{~mm}$ thick wafers. The first one has the holes for the injection and removing of the liquids (inlets and outlets) and the second one includes the channels. The die comprises two channels with the width of 1 and $6 \mathrm{~mm}$ long. One is needed to obtain the baseline reference and to calibrate the light source. The other allows the mixed solution analysis (reagent plus sample). The transmitted light through the mixture is measured by photodetectors placed underneath both channels in the CMOS die.

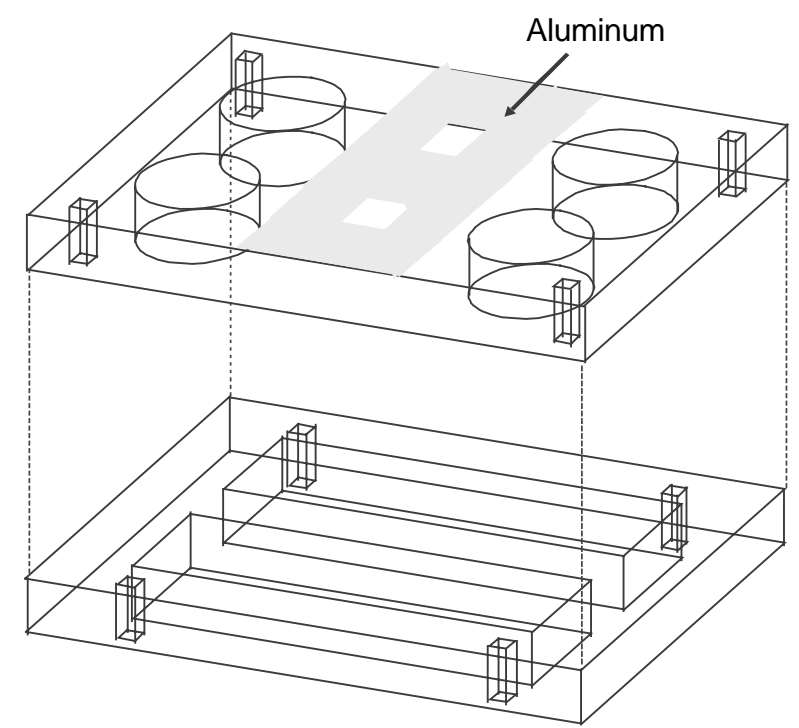

Fig. 2. Polystyrene die.

\subsection{CMOS die}

The photodetectors and the readout electronics are integrated in the same die. The photodetectors are made using the layers available in a CMOS standard process only and without additional masks or steps. Fortunately, in the visible part of the spectrum, the absorption of light in silicon is wavelength dependent and the response can be programmed by design. A deeper junction shows in general an enhanced long-wavelength response. Selecting the suitable junction depth allows to set a maximum peak at a specific wavelength [5]. In addition, the spectral response is determined by the thickness and material properties of the dielectric layers available in the CMOS standard process. The arrangement of those different layers forms an interference filter with the interference peak at different wavelengths. Therefore, the maximum obtainable spectral selectivity highly depends on the process used.

The photodetectors are pn-junction photodiodes fabricated using a p-substrate/n-diffusion junction. This structure is chosen, because it has the higher quantum efficiency in the desired spectral range (as compared to different types of photodiodes available in a CMOS standard process) [6].

It is desirable to integrate the analog to digital conversion with the light sensors. The A/D conversion is performed by using a one-bit first-order sigma delta modulator. A block diagram of the light sensors with the A/D conversion is shown in Fig. 3. The circuit consists of the following sections: a current difference amplifier; an integrator and a 1-bit A/D converter in the forward path; and a 1-bit D/A converter in the feedback path of a single feedback loop system. The current difference amplifier generates the input value of the converter by the difference in the photocurrents measured by the two photodiodes. The integrator provides the delay 


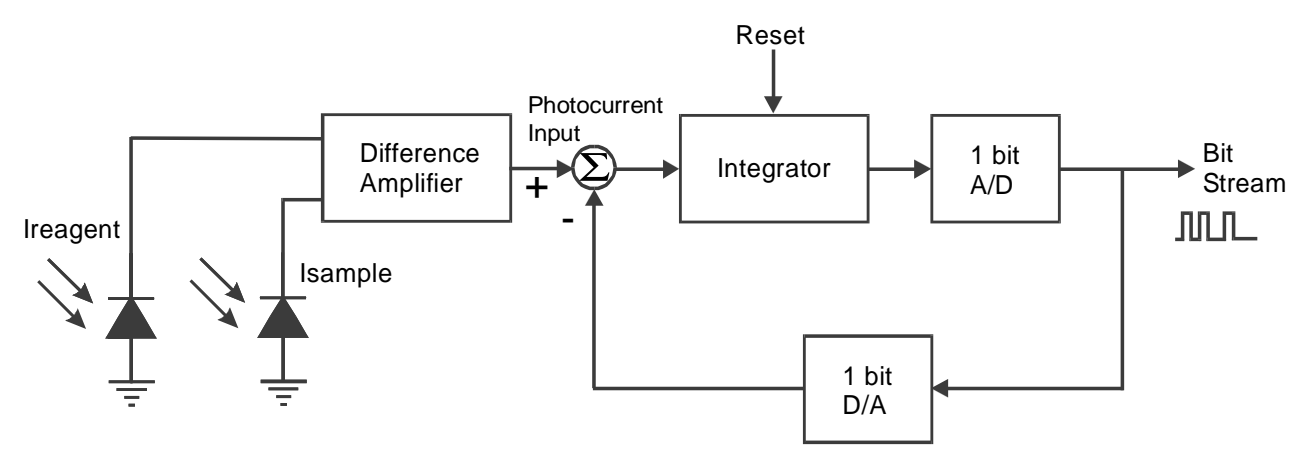

Fig. 3. Block diagram of the readout circuit.

needed. The 1-bit A/D converter is a latched comparator that converts an analog signal into either a high or a low. The 1-bit D/A converter (two voltage-controlled switches) uses the comparator output to determine if a high or a low is summed with the input. Therefore, the bio-system has a bit stream output and allows its use in small data-acquisition and control systems. That bit stream is proportional to the light intensity transmitted through the mixture.

\section{Bio-system fabrication}

\subsection{Polystyrene die}

In the polystyrene die, holes and channels are drilled and milled, respectively, by using a CNC machine. Then both wafers are glued. An aluminum layer is deposited on top of the wafer, acting as a light shield between the channels (Fig. 2). Polystyrene is chosen because of its optical transparency in the visible part of the spectra and because it is a good insulator and a suitable material to drill holes when compared with glass. Therefore, the electrophoretic flow principle can be used to move fluids through the microchannels, which avoids mechanical pumps and valves.

\subsection{CMOS die}

The CMOS compatible photodetectors and readout electronics of the colorimetric detection system are fabricated through a double-metal, single-polysilicon, $1.6 \mu \mathrm{m} n$-well CMOS standard process. The photodiodes have an active area of $500 \mu \mathrm{m} \times 500 \mu \mathrm{m}$ each.

The basic structure of the photodetector is shown in Fig. 4. The thickness of the $n+$ layer is $350 \mathrm{~nm}$ and the depth of the epilayer is $12 \mu \mathrm{m}$, with a doping concentration of $10^{16}$ atoms $/ \mathrm{cm}^{3}$. The first oxide (BPSG) thickness above the diode is measured as $650 \mathrm{~nm}$ and the second $\left(\mathrm{SiO}_{2}\right)$ as $700 \mathrm{~nm}$. The silicon nitride layer, used for scratch protection, is $800 \mathrm{~nm}$ thick. Since technology rules from the CMOS standard process have to be met, the design of the optical path is restricted to combinations of those three dielectric layers above the pn-junction. The simulated optical trans-



Fig. 4. Cross-section of the fabricated CMOS compatible photodetector.

missions of those combinations are shown in Fig. 5. The simulations are done with an optics software package TFCalc 3.4, supplied by Software Spectra, Inc., USA. Simulations indicate that an oxide layer, with a thickness around $700 \mathrm{~nm}$, can increase the transmittance from 50 to $60 \%$, but also introduces wavelength dependence. However, Fig. 6 shows that the removal of the first oxide layer causes bubbles in the photodiode active area. This random surface-rough interferes, also randomly, in the photocurrent response. Therefore, using the CMOS standard process, the first oxide layer should be left over (Fig. 4). Fig. 7 shows the spectral response curve of the fabricated photodiode, measured using the experimental setup described in Section 4 and a calibrated Hamamatsu S1336-5BQ photodiode as a reference. The complete device is shown in Fig. 8. The polystyrene die is glued to the CMOS chip.

\section{Measurement setup}

The experimental arrangement used in the measurements comprises a $150 \mathrm{~W}$ Xe lamp with a monochromator TRIAX-180 $(1200 \mathrm{~g} / \mathrm{mm}$ grating with a spectral dispersion of $3.6 \mathrm{~nm} / \mathrm{mm}$ and a spectral resolution of $0.3 \mathrm{~mm}$ at $546 \mathrm{~nm}$ ) that was used as light source and a Keithley 487 picoammeter (full-scale range from $10 \mathrm{fA}$ to $2 \mathrm{~mA}$ and a resolution of $5^{1 / 2}$ digit) for measuring the photodiode current. Instead of the monochromatic light source, white light could be used. In this case, an optical filter should be placed on the top of the photodetectors to select a wavelength range. Macroscopic measurements (Fig. 1) show that a 


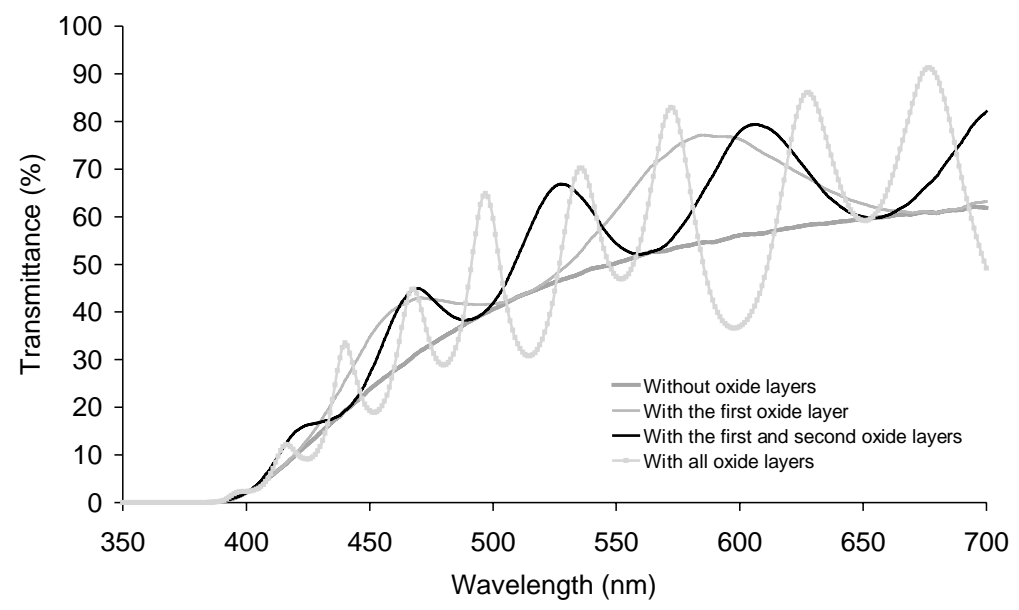

Fig. 5. Simulated spectral responses of some typical combinations of dielectric layers (available in the CMOS standard process) above the silicon.

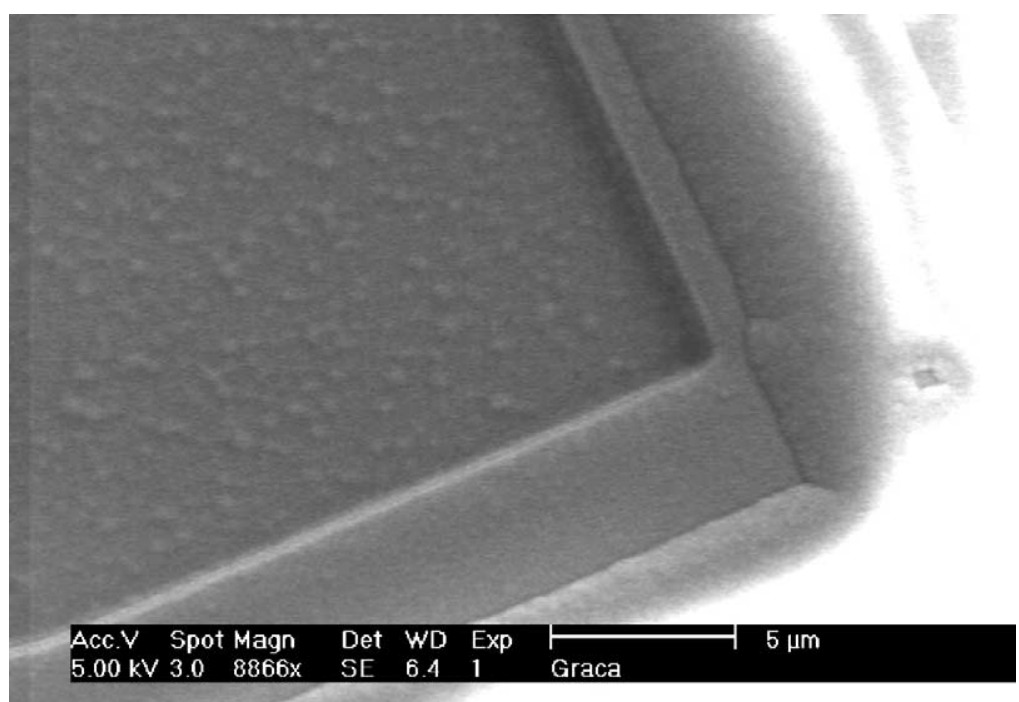

Fig. 6. An SEM picture of the CMOS photodiode without the first oxide shows the random surface roughness.



Fig. 7. Measured spectral response of the fabricated photodiode.



Fig. 8. Packaged bio-system. 
rough band-pass optical filter is sufficiently selective, since the FWHM of the absorption spectra is $90 \mathrm{~nm}$.

\section{Experimental results}

The reagent used in the measurements was the infinity ${ }^{\mathrm{TM}}$ uric acid reagent from Sigma-Aldrich. It contains approximately $0.5 \mathrm{mmol} / \mathrm{l}$ 4-aminoantipyrine, $1.75 \mathrm{mmol} / \mathrm{l} \mathrm{TBHB}$, $>32$ units/l uricase (bacillus sp.), >1300 units/l peroxidase (horseradish), buffer $\mathrm{pH} 8.0$, and $0.05 \%$ sodium azide as preservative [7]. This reagent reacts with a sample of urine containing uric acid in a 50:1 ratio, and produces an absorption maximum at a specific wavelength $(\lambda=494 \mathrm{~nm})$. Studies of the influence of some main substances found in urine on the determination of uric acid were carried out. Bilirubin (free and conjugated), cysteine, glucose, hemoglobin or sodium did not interfere significantly with the analytical procedure. Hemoglobin and bilirubin inhibited only in high concentration $(>200 \mathrm{mg} / \mathrm{dl}$ for hemoglobin, $>8 \mathrm{mg} / \mathrm{dl}$ for free bilirubin and $>12 \mathrm{mg} / \mathrm{dl}$ for conjugated bilirubin, for example). Young [8] has published a comprehensive list of drugs and substances that may interfere with this assay.

Fig. 9 shows the measured transmittance response for different uric acid concentrations in urine. Measurement results are done from 5 to $120 \mathrm{mg} / \mathrm{dl}$, comprising the range of normal and usually abnormal values in a human being $(17-67 \mathrm{mg} / \mathrm{dl})$. Concentration differences less than $5 \mathrm{mg} / \mathrm{dl}$ are not taken into account, since it means a variation of less than $10 \%$ in the values typical for a human being, as above-mentioned. The transmittance is defined as $T=I / I_{0}$, where $I$ is the measured photodiode current for each solution and $I_{0}$ the measured photodiode current of the reagent. The transmittance at $\lambda=494 \mathrm{~nm}$ as a function of the different uric acid concentrations is shown in Fig. 10. The results obtained agree with the macroscopic measurements and the same conclusions can be achieved.

Table 1 gives some useful calculations based on the measured values. The absorption coefficient for each concentra-



Fig. 9. Measured transmittance spectra for different uric acid concentrations. From top to bottom curve: reagent, 5, 10, 15, 20, 30, 40, 60, 80 and $120 \mathrm{mg} / \mathrm{dl}$.

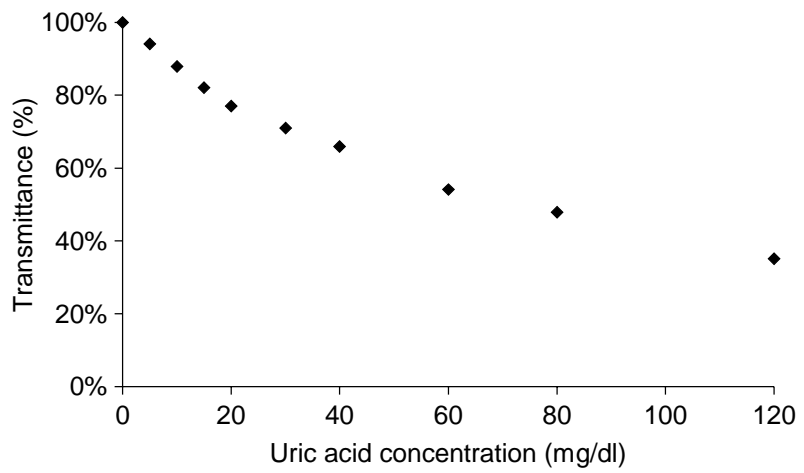

Fig. 10. Transmittance at $\lambda=494 \mathrm{~nm}$ for the measured uric acid concentrations.

Table 1

Absorption coefficient of each measured concentration for $\lambda=494 \mathrm{~nm}$ and for $\lambda=360 \mathrm{~nm}$ (last column)

\begin{tabular}{lccccc}
\hline Solutions & $I(\mu \mathrm{A})$ & $\alpha\left(\mathrm{m}^{-1}\right)$ & $I / I_{0}$ & $T_{\text {diff }}$ & $\alpha\left(\mathrm{m}^{-1}\right)$ \\
\hline Reagent & 0.177 & 0 & 1.00 & & 0 \\
$5 \mathrm{mg} / \mathrm{dl}$ & 0.167 & 54.97 & 0.94 & 0.06 & 11.10 \\
$10 \mathrm{mg} / \mathrm{dl}$ & 0.155 & 117.68 & 0.88 & 0.06 & 28.03 \\
$15 \mathrm{mg} / \mathrm{dl}$ & 0.145 & 179.42 & 0.82 & 0.06 & 37.92 \\
$20 \mathrm{mg} / \mathrm{dl}$ & 0.141 & 208.64 & 0.79 & 0.03 & 46.58 \\
$30 \mathrm{mg} / \mathrm{dl}$ & 0.126 & 311.95 & 0.71 & 0.08 & 82.93 \\
$40 \mathrm{mg} / \mathrm{dl}$ & 0.117 & 378.49 & 0.66 & 0.05 & 117.61 \\
$60 \mathrm{mg} / \mathrm{dl}$ & 0.096 & 557.75 & 0.63 & 0.03 & 148.50 \\
$80 \mathrm{mg} / \mathrm{dl}$ & 0.085 & 670.15 & 0.48 & 0.15 & 165.37 \\
$120 \mathrm{mg} / \mathrm{dl}$ & 0.062 & 951.65 & 0.35 & 0.13 & 248.17 \\
\hline
\end{tabular}

tion $(\alpha)$ was calculated by the Beer-Lambert law:

$I_{\lambda}(\mathrm{LP})=I_{\lambda}(\mathrm{LP}=0) \mathrm{e}^{-\alpha_{\lambda} \mathrm{LP}}$

In the fifth column it can be seen that the minimum transmittance difference $\left(T_{\text {diff }}\right)$ between successive measured concentrations $(5 \mathrm{mg} / \mathrm{dl})$ is $3 \%$. However, the relative sensitivity of this device is $0.33 \mathrm{mg} / \mathrm{dl}(0.4 \%)$, with a $1 \mathrm{~mm}$ lightpath. Thus, an 8-bit analog-to-digital converter in the readout electronics provides a sufficient resolution for this sensitivity and it determines the detection limit, in the presented prototype.

The sensitivity achieved with the bio-system is suitable for human being urine values. Therefore, the lightpath can be decreased (the sensitivity will also decrease, but the resolution of the $\mathrm{A} / \mathrm{D}$ converter is maintained). A sensitivity of $3.65 \mathrm{mg} / \mathrm{dl}$ ( $7.3 \%$ of human being urine values) can be achieved with a lightpath of $100 \mu \mathrm{m}$ in a $500 \mu \mathrm{m}$ Pyrex wafer.

\section{Conclusions}

A small-size and low-cost bio-system for real-time measurement of uric acid in a urine sample is presented in this paper. The sensitivity obtained with the optical absorption method (with a lightpath of $1 \mathrm{~mm}$ ), is enough to 
give a $\pm 0.6 \%$ resolution for human being urine values. The achieved sensitivity is $0.33 \mathrm{mg} / \mathrm{dl}$. This detection system avoids the need of expensive readout optics and opens the door to low-cost disposable devices. Moreover, tests using ambient light have been done to avoid the use of a known source of light. Although this microsystem is presented for urine analysis, other biological fluids (such as serum, sweat, saliva or cerebrospinal fluid) are potential candidates for the bio-system.

Other applications for the bio-system are: monitoring of air and water quality (looking for toxins and pesticides, etc.) and fast identification of drugs abuse. Bio-system devices will probably find their way into forensic, environmental and food testing laboratories in the near future. Moreover, since low quantities of hazardous chemical reagents are needed, the resultant environmental pollution is negligible.

\section{Acknowledgements}

The authors wish to acknowledge the cooperation of Ger de Graaf from the Laboratory for Electronic Instrumentation, TUDelft, The Netherlands. This work is supported by the Portuguese Foundation of Science and Technology (FCT projects SFRH/BD/1281/2000 and POCTI/33747/ESE/1999) and by FEDER.

\section{References}

[1] S.C. Jakeway, A.J. de Mello, E. Russell, Miniaturized total analysis systems for biological analysis, Fresenius J. Anal. Chem. 366 (2000) $525-539$.

[2] M.U. Kopp, H.J. Crabtree, A. Manz, Developments in technology and applications of microsystems, Curr. Opin. Chem. Biol. 1 (1997) $410-419$.

[3] A.J. Tüdõs, G.A.J. Besselink, R.B.M. Schasfoort, Trends in miniaturized total analysis systems for point-of-care testing in clinical chemistry, Lab-on-a-Chip 1 (2) (2001) 83-95.

[4] J.P. Peters, D.D. Van Slyke, Quantitative Clinical Chemistry Interpretations, vol. 1, Williams and Wilkins, Baltimore, 1946.

[5] H.R. Philipp, E.A. Taft, Optical constants of silicon in the region 1 to 10 ev, Phys. Rev. 120 (1) (1960) 37-38.

[6] B. Fowler, A. El Gamal, D.X.D. Yang, A CMOS area image sensor with pixel-level A/D conversion, ISSCC Digest of Technical Papers, San Francisco, CA, February 1994.
[7] P. Kabasakalian, S. Kalliney, A. Westcott, Determination of uric acid in serum, with use of uricase and a tribromophenol-aminoantipyrine chromogen, Clin. Chem. 19 (5) (1973) 522-524.

[8] D.S. Young, Effects of Drugs on Clinical Laboratory Tests, vol. 3, 3rd ed., American Association for Clinical Chemistry, 1990, pp. 360-370.

\section{Biographies}

G. Minas graduated in industrial electronics engineering in 1994 and obtained her MSc degree in 1998 both titles at University of Minho, Portugal. Since 1995 she has been a Lecturer in Department of Industrial Electronics, University of Minho, Portugal and she is involved in biomedical microdevices research.

J.S. Martins graduated in electrical engineering at University of Oporto, Portugal in 1979. He obtained his PhD degree in computer engineering at University of Minho, Portugal in 1993. Since 1998, he has been an Associate Professor at the Department of Industrial Electronics of the University of Minho and he is involved in electronics and instrumentation research

R.F. Wolffenbuttel received his $\mathrm{MSc}$ and $\mathrm{PhD}$ degrees from the Delft University of Technology, Delft, The Netherlands, in 1984 and 1988, respectively. Between 1986 and 1993, he was an Assistant Professor, and since 1993, an Associate Professor, at the Laboratory of Electronic Instrumentation of the Delft University of Technology, where he is involved in instrumentation and measurement in general and on-chip integration of microelectronic circuits and silicon sensor, fabrication compatibility issues, and micromachining in silicon and microsystems, in particular. $\mathrm{He}$ was a Visitor at the University of Michigan, Ann Arbor, in 1992, 1999, and 2001, at Tohoku University, Sendai, Japan, in 1995, and at EPFL, Lausanne, Switzerland, in 1997. He was the recipient of a 1997 NOW pioneer award. He served as General Chairman of the Dutch National Sensor Conference in 1996 and Eurosensors in 1999.

J.H. Correia graduated in physical engineering at University of Coimbra, Portugal in 1990. He obtained his PAPCC (equivalent to MSc degree) in 1994 at University of Minho, Portugal and his PhD degree in 1999 at Delft University of Technology, The Netherlands. His thesis work dealt with the development of optical microsystems in silicon for the visible part of the spectrum. Since May 1999 he has been an Assistant Professor at the Department of Industrial Electronics of the University of Minho and he is involved in instrumentation and measurement in general and on-chip functional integration of microelectronic circuits and silicon sensors.

J.C. Ribeiro graduated in Electrical Engineering at University of Minho, Portugal in 2003. Since September 2002, he is working in the microelectronics group at University of Minho, Portugal and he is involved in biomedical microdevices research. 